\title{
Impact of somatic PI3K pathway and ERBB family mutations on pathological complete response $(p C R)$ in HER2-positive breast cancer patients who received neoadjuvant HER2-targeted therapies
}

Sinead Toomey ${ }^{1 *+}\left(\mathbb{D}\right.$, Alexander J. Eustace ${ }^{1 \dagger}$, Joanna Fay ${ }^{2}$, Katherine M. Sheehan ${ }^{2}$, Aoife Carr ${ }^{1}$, Malgorzata Milewska', Stephen F. Madden ${ }^{1}$, Ausra Teiserskiene ${ }^{3}$, Elaine W. Kay ${ }^{2}$, Norma O'Donovan ${ }^{4}$, William Gallagher ${ }^{5}$, Liam Grogan ${ }^{6}$, Oscar Breathnach ${ }^{6}$, Janice Walshe', Catherine Kelly ${ }^{8}$, Brian Moulton, M. John Kennedy ${ }^{9}$, Guiseppe Gullo ${ }^{7}$, Arnold D. Hill ${ }^{10}$, Colm Power $^{10}$, Deirdre Duke ${ }^{11}$, Niamh Hambly ${ }^{11}$, John Crown ${ }^{4,7 \dagger}$ and Bryan T. Hennessy ${ }^{1,3,6+}$

\footnotetext{
Abstract

Background: The Cancer Genome Atlas analysis revealed that somatic EGFR, receptor tyrosine-protein kinase erbB-2 (ERBB2), Erb-B2 receptor tyrosine kinase 3 (ERBB3) and Erb-B2 receptor tyrosine kinase 4 (ERBB4) gene mutations (ERBB family mutations) occur alone or co-occur with somatic mutations in the gene encoding the phosphatidylinositol 3-kinase (PI3K) catalytic subunit (PIK3CA) in 19\% of human epidermal growth factor receptor 2 (HER2)-positive breast cancers. Because ERBB family mutations can activate the PI3K/AKT pathway and likely have similar canonical signalling effects to PI3K pathway mutations, we investigated their combined impact on response to neoadjuvant HER2-targeted therapies.

Methods: Baseline tumour biopsies were available from 74 patients with HER2-positive breast cancer who were enrolled in the phase II TCHL neoadjuvant study (ICORG 10-05) assessing TCH (docetaxel, carboplatin, trastuzumab) $(n=38)$ versus TCL (docetaxel, carboplatin, lapatinib) $(n=10)$ versus TCHL (docetaxel, carboplatin, trastuzumab, lapatinib) ( $n=40)$, each for six cycles. Activating mutations in PIK3CA and ERBB family genes were identified using mass spectrometry-based genotyping. Phosphatase and tensin homolog (PTEN) expression was assessed by immunohistochemistry.

(Continued on next page)
}

\footnotetext{
* Correspondence: sineadtoomey@rcsi.ie

${ }^{\dagger}$ Equal contributors

${ }^{1}$ Medical Oncology Group, Department of Molecular Medicine, Royal College

of Surgeons in Ireland, Dublin 9, Ireland

Full list of author information is available at the end of the article
} 
(Continued from previous page)

Results: PIK3CA and/or ERBB family mutations were detected in 23 (31.1\%) tumour samples tested, whereas PTEN expression was low in $31.1 \%$ of cases tested. Mutation frequency was similar in each treatment arm (31.3\% in TCH arm, 30\% in TCL arm and 31.3\% in TCHL arm) and was not influenced by oestrogen receptor (ER) status (27.6\% in ER-negative patients, $33.3 \%$ in ER-positive patients) or progesterone receptor (PR) status (32.6\% in PR-negative patients, 29\% in PR-positive patients). There was no significant difference in pathological complete response (pCR) rates between 47 patients with wild-type (WT) tumours and 22 patients whose tumours carried mutations (in either PIK3CA or ERBB family genes) (42.5\% vs. $54.5 \%$; $p=0.439)$. Similarly, there was no significant difference in pCR rates between patients with PIK3CAVERBB family mutated/PTEN-low (i.e., PI3K-activated) tumours and patients without PI3K activation (50\% vs. 44\%; $p=0.769$ ). However, in the TCHL (but not the TCH) group, the pCR rate was higher for 9 patients with PIK3CA/ERBB family mutated tumours than for 20 patients with PIK3CA/ERBB family WT tumours (77.8\% vs. 35\%; $p=0.05)$.

Conclusions: Our results indicate that patients who receive neoadjuvant TCHL and have PIK3CA/ERBB family mutated tumours may be more likely to have a pCR than patients with WT tumours.

Trial registration: ClinicalTrials.gov, NCT01485926. Registered on 2 December 2011.

Keywords: Breast cancer, HER2, Trastuzumab, Lapatinib, PI3K pathway, Somatic mutations

\section{Background}

Approximately $20 \%$ of primary breast cancers have an alteration, usually amplification, of the human epidermal growth factor receptor 2 (HER2) gene. HER2-amplified cancers have an inferior prognosis, with more frequent and more rapid metastatic relapse [1]. The addition of trastuzumab to conventional chemotherapy has significantly improved the outcomes for patients with both early-stage and metastatic HER2-altered breast cancer, with significantly improved survival in both settings [2]. Although a small minority of patients with HER2-positive metastatic disease achieve durable complete remissions, approximately $80 \%$ to $90 \%$ develop progressive cancer. Addition of trastuzumab to pre-operative chemotherapy results in $30 \%$ to $50 \%$ of patients achieving a pathological complete response (pCR) [2]. Despite this, approximately $20 \%$ of patients with HER2-positive early-stage disease and $90 \%$ of patients with metastatic disease will die from disease which is resistant to trastuzumab. Newer targeted antiHER2 agents have been studied in an attempt to overcome trastuzumab resistance [3-5].

One such agent is lapatinib, a small-molecule inhibitor of epidermal growth factor receptor (EGFR) and receptor tyrosine-protein kinase erbB-2 (ERBB2). In a large randomised trial, the addition of lapatinib to capecitabine in patients whose cancer had progressed following treatment with anthracycline, taxanes and trastuzumab was associated with statistically prolonged progression-free survival compared with capecitabine alone [6]. Evidence suggests that combined targeting of both the extracellular domain of HER2 with trastuzumab and the kinase domain with lapatinib may further improve response. We have shown that combined treatment with trastuzumab and lapatinib improves response to chemotherapy in SKBR3 breast cancer cells and decreases tumour growth in BT-474 xenografts [7], with the combination also showing improved response in the neoadjuvant treatment of HER2-positive breast cancer in some clinical trials [8-11]. However, the benefit of combined HER2-targeted therapy has not been shown consistently. The National Surgical Adjuvant Breast and Bowel Project protocol B-41 demonstrated no significant difference in $\mathrm{pCR}$ rates between patients receiving the combination of trastuzumab and lapatinib and those receiving either single agent [12], while the EORTC 10054 study demonstrated a numerically higher but nonsignificant benefit of double anti-HER2 blockade with trastuzumab and lapatinib [13]. Thus, to determine for ourselves whether the addition of lapatinib to, or the substitution of lapatinib for, trastuzumab would improve pCR, we initiated protocol ICORG 10-05, a prospective, randomised trial with stage Ic/II/III HER2-positive breast cancer patients (NCT01485926) [14].

Activating somatic mutations in the phosphatidylinositol 3-kinase (PI3K)/AKT pathway are present in a range of tumour types $[15,16]$. Mutations in PIK3CA occur in approximately one-third of breast cancers [17], and these mutations have been implicated in the development of trastuzumab resistance $[18,19]$. Ligand binding to ERBB family members activates intracellular signalling pathways such as the PI3K/AKT pathway [20]. Trastuzumab and lapatinib block this signalling, either by binding ERBB2 at the cell surface or by directly inhibiting the kinase activity of both EGFR and ERBB2 [20]. Possible resistance mechanisms include constitutive activation of the PI3K/AKT pathway through somatic mutations in the PI3K pathway or altered intracellular signalling involving loss of phosphatase and tensin homolog (PTEN) [18]. 
Mutations in EGFR, ERBB2, Erb-B2 receptor tyrosine kinase $3(E R B B 3)$ and Erb-B2 receptor tyrosine kinase 4 (ERBB4) (referred to hereinafter as $E R B B$ family mutations) either occur alone or co-occur with PIK3CA mutations in 19\% of HER2-positive breast cancers $(n=58)$ [21, 22]. Because ERBB-mediated effects are dependent on PI3K/AKT signalling and $E R B B$ family mutations can activate the PI3K/AKT pathway, it is likely that they have similar canonical signalling effects to PI3K pathway mutations and PTEN loss. Therefore, the primary aim of our study was to investigate the association of $\mathrm{pCR}$ with $E R B B$ family and PIK3CA mutations and PTEN loss (defined as PI3K pathway activation) in primary HER2-positive breast cancer treated with one or two HER2-targeting agents.

\section{Methods}

\section{Patient population and samples}

TCHL (ICORG10-05) (NCT01485926) is a phase II neoadjuvant study assessing $\mathrm{TCH}$ (docetaxel, carboplatin, trastuzumab), TCL (docetaxel, carboplatin, lapatinib) and TCHL (docetaxel, carboplatin, trastuzumab, lapatinib) in stages Ic-III HER2-positive breast cancer patients. Full details of the trial are available at www.clinicaltrials.gov. Eighty-eight patients were randomised to receive either neoadjuvant $\mathrm{TCH}$ (six cycles q3weekly (every 3 weeks) docetaxel $\left[75 \mathrm{mg} / \mathrm{m}^{2}\right]+$ carboplatin [AUC 6] + trastuzumab $8 \mathrm{mg} / \mathrm{kg}$ on day 1 [loading dose] and $6 \mathrm{mg} / \mathrm{kg}$ for subsequent cycles, q3weekly (every 3 weeks)), TCL (six cycles q3weekly (every 3 weeks) docetaxel $\left[75 \mathrm{mg} / \mathrm{m}^{2}\right]$ + carboplatin [AUC 6] + lapatinib [1000 mg daily until 1 week prior to surgery]) or TCHL (six cycles q3weekly (every 3 weeks) docetaxel $\left[75 \mathrm{mg} / \mathrm{m}^{2}\right]+$ carboplatin [AUC 6] + trastuzumab $8 \mathrm{mg} / \mathrm{kg}$ on day 1 [loading dose] and $6 \mathrm{mg} / \mathrm{kg}$ for subsequent cycles [q3weekly (every 3 weeks)] + lapatinib [1000 mg daily until 1 week prior to surgery]). Patients subsequently underwent surgery and received trastuzumab post-operatively for 1 year from the first dose of trastuzumab. The primary endpoint of the trial was to assess the efficacy of TCH, TCL and TCHL in the neoadjuvant treatment of HER2-positive breast cancer using pCR. Secondary objectives were to assess the clinical response rate and overall response rate in each treatment arm in HER2-positive breast cancer patients and to examine potential molecular and pharmacological markers of response to trastuzumab- and lapatinib-based chemotherapy.

\section{Sample processing and MassARRAY analysis}

Baseline tumour biopsies obtained prior to neoadjuvant chemotherapy were fixed in formalin and embedded in paraffin wax (FFPE). Haematoxylin and eosin staining was performed on 3- $\mu \mathrm{m}$ sections of biopsies and assessed for tumour cellularity by a pathologist. Only samples with greater than $10 \%$ tumour cellularity were used for further analysis. DNA extraction was performed using an AllPrep ${ }^{\mathrm{Tm}}$
DNA/RNA Mini Kit (QIAGEN, Hilden, Germany) as per the manufacturer's instructions. Mass spectrometry-based single-nucleotide polymorphism genotyping technology (Agena Bioscience, San Diego, CA, USA) was applied to DNA extracted from the FFPE biopsies to detect a total of 108 non-synonymous somatic mutations in PIK3CA, $E G F R, E R B B 2, E R B B 3$ and ERBB4. Hot spot mutations in exon 1 (R88Q, K111N), exon 4 (N345K), exon 7 (C420R, E453K), exon 9 (E542V/G/K/Q, E545K/Q/D/A/G/V, Q546 $\mathrm{H} / \mathrm{L} / \mathrm{P} / \mathrm{R} / \mathrm{E} / \mathrm{K})$ and exon 20 (Y1021H/N/C, R1023Q, T102 5I/A/S, A1035V/T, M1043V/I, A1046V, H1047R/L/Y, G104 9R) of PIK3CA were analysed. Mutations in ERBB family genes were identified using publicly available data from The Cancer Genome Atlas database and a literature search [22]. AVSIFT and Mutation Assessor scores were used to determine the $E R B B$ family mutations that were likely to be deleterious. A full list of mutations is provided in Additional file 1: Table S1. Matrix chips were analysed on a MassARRAY matrix-assisted laser desorption/ionisation time-offlight mass spectrometry system (Agena Bioscience). Visual inspection and Typer Software were used to identify genotypes on the basis of mass spectra. Reactions where greater than $15 \%$ of the resultant mutant mass ran in the mutant site were scored as positive.

\section{PTEN immunohistochemistry}

PTEN immunohistochemistry was performed on 4- $\mu \mathrm{m}$ sections of FFPE tumour biopsies as described previously [23]. Briefly, tissue sections were deparaffinised and rehydrated prior to antigen retrieval at $100{ }^{\circ} \mathrm{C}$ for 20 minutes with Bond Epitope Retrieval Solution 1 (Leica Biosystems, Newcastle upon Tyne, UK). Endogenous peroxidase was blocked with $3 \%$ peroxidase for 5 minutes. PTEN antibody (clone 6H2.1; Dako/Agilent Technologies, Glostrup, Denmark) was applied at 1:100 dilution, and primary antibody detection was carried out using a polymer system (Bond Polymer Refine Detection; Leica Biosystems). Staining development was achieved by incubation with 3,3'-diaminobenzidine (DAB) and DAB enhancer (Leica Biosystems). PTEN immunohistochemistry was scored by a pathologist as follows. PTEN was considered absent (score $=0$ ) if no immunostaining was detectable in breast carcinoma cells but was present in adjacent benign stromal cells (which acted as an internal control), scored as $1+$ if cytoplasmic immunostaining was weak, as $2+$ if cytoplasmic immunostaining in carcinoma cells was intermediate, and as $3+$ if the cytoplasmic immunostaining in breast carcinoma cells was strong.

\section{Statistical analysis}

Differences in pCR rates by mutation and PTEN status were calculated with a corresponding 95\% CI and tested using a $x^{2}$ test of association. The treatment effect was further examined by performing $\chi^{2}$ tests of association 
on $\mathrm{pCR}$ and mutation status and PTEN status for each treatment arm separately.

\section{Results \\ Patient characteristics}

Eighty-eight patients were enrolled in the TCHL clinical trial [14]. The median age of the patients at diagnosis was 49 years. Of the 88 patients $53(60.2 \%)$ had oestrogen receptor (ER)-positive tumours, whereas $34(38.6 \%)$ had progesterone receptor (PR)-positive tumours. Thirty-eight (43.2\%) of the patients had a pCR (defined as no residual invasive tumour in the breast or lymph nodes at surgery). Also, $38(43.2 \%)$ of the patients received trastuzumab $(\mathrm{TCH})$, whereas $40(45.4 \%)$ of the patients received the combination of lapatinib and trastuzumab (TCHL). Only $10(11.4 \%)$ of the patients received lapatinib alone (TCL), because this arm of the trial was closed early owing to preliminary data emerging from the Adjuvant Lapatinib and/or Trastuzumab Treatment Optimisation (ALTTO) study indicating that TCL treatment was inferior to TCH or TCHL in terms of patient survival outcomes [24]. The full clinical characteristics of the patient cohort are detailed in Table 1.

\section{Somatic mutation profiling}

Each biopsy taken prior to neoadjuvant chemotherapy was assessed for tumour cellularity by a pathologist, and samples with less than $10 \%$ tumour material or that produced low DNA yield were excluded from the analysis, which left a total of 74 tumour samples for sequencing analysis. MassARRAY analysis was used to identify hot spot mutations in PIK3CA, EGFR, ERBB2, ERBB3 and ERBB4. PIK3CA mutations alone were detected in 15 breast tumour samples (20.3\%), whereas EGFR mutations alone were detected in 2 samples (2.7\%). ERBB3 and ERBB4 mutations alone were each detected in one sample (1.4\%). No activating mutations were detected in ERBB2. EGFR, $E R B B 3$ and ERBB4 mutations co-occurred with PIK3CA mutations in 1 sample each (1.4\%), whereas 1 sample had co-occurring ERBB3 and ERBB4 mutations (1.4\%) (Fig. 1), giving an overall PIK3CA and/or ERBB mutation frequency of $31.1 \%$ (23 samples). This overall mutation frequency was similar in each treatment arm $(30 \%$ in TCL arm [three samples], $31.3 \%$ in $\mathrm{TCH}$ arm [ten samples], and $31.3 \%$ in TCHL arm [ten samples]) and was not influenced by ER status (27.6\% in ER-negative tumours, $33.3 \%$ in ER-positive tumours) or PR status (32.6\% in PR-negative tumours, 29\% in PR-positive tumours) (Table 2). Specific mutations identified are detailed in Additional file 2.

PTEN expression and its overlap with mutation status We examined the expression of PTEN protein by immunohistochemical analysis in the diagnostic biopsies of 45 tumours where there was sufficient tissue for PTEN evaluation. Representative staining patterns for each parameter are shown in Fig. 2a. Fourteen (31.1\%) of 45 tumours were considered to have low (absent or weak) PTEN expression $(0 / 1+)$ in the tumour (Fig. 2b). No differences in expression rates were seen among the three different treatment arms (Table 3). Low PTEN expression, although less frequent in cases with concurrent PIK3CA/ERBB mutations (10 [22.2\%] of 45 vs. 4 [8.9\%] of 45), was not mutually exclusive with PIK3CA/ERBB mutations (Fig. 2c). Low PTEN expression was more frequently seen in PR-negative than in PR-positive tumours (37.9\% vs. $18.8 \%$ ); however, there was no difference in the frequency of low PTEN expression between ER-negative and ER-positive tumours (31.6\% vs. 30.8\%) (Table 3). PI3K pathway activation (defined by a PIK3CA/ERBB mutation and/or low expression of PTEN) was found in 25 (55.6\%) tumours overall and was not influenced by either ER or PR status: $57.9 \%$ of ER-negative tumours and $53.8 \%$ of ERpositive tumours had an activated PI3K pathway, whereas $58.6 \%$ of PR-negative and $50 \%$ of PR-positive tumours had an activated PI3K pathway. PI3K pathway activation also occurred at similar frequencies in each treatment arm $(66.7 \%$ in TCL arm [4 samples], $61.9 \%$ in TCH arm [13 samples] and $44.4 \%$ in TCHL arm [8 samples]) (Table 3).

\section{Mutation and PTEN status and $\mathrm{pCR}$}

In the combined TCH/TCHL study population, we analysed the association between tumour mutation status and $\mathrm{pCR}$, as defined by no invasive cancer in the breast and no involvement of axillary nodes in 69 patients where pCR data were available (Fig. 3). There was no significant difference in $\mathrm{pCR}$ rates between 17 patients with PIK3CA mutated vs. 52 patients with PIK3CA wildtype (WT) tumours ( $47 \%$ vs. $46.1 \%$; $p=1.000)$ or between 8 patients with $E R B B$ family mutated vs. 61 patients with $E R B B$ family WT tumours (62.5\% vs. $47.5 \%$; $p=0.477)$. When we combined tumours with PIK3CA and ERBB family mutations, there was no difference in pCR rates between 22 patients with mutated vs. 47 patients with WT tumours ( $54.5 \%$ vs. $42.6 \% ; p=0.439)$ (Fig. 3 ). We also found no difference in $\mathrm{pCR}$ rates between 14 patients with low tumour PTEN expression and 31 patients with moderate or strong PTEN expression in their tumours (42.9\% vs. $48.4 \% ; p=0.759$ ). When we combined tumours with a PIK3CA/ERBB family mutation and/or PTEN loss (i.e., PI3K pathway activated), we found no difference in $\mathrm{pCR}$ rates between 25 patients with PI3K activated tumours and 20 patients with tumours without an activated PI3K pathway ( $44 \%$ vs. $50 \%$; $p=0.769$ ) (Fig. 3 ).

There was no difference in overall pCR rates in each treatment arm (Table 4); however, we also specifically examined the influence of mutation status on $\mathrm{pCR}$ in 
Table 1 Patient clinical characteristics

\begin{tabular}{|c|c|c|c|c|c|c|}
\hline \multirow[b]{2}{*}{ Characteristic } & \multicolumn{2}{|c|}{ TCHL study $(n=88)$} & \multicolumn{2}{|c|}{ Sequencing and pCR data available $(n=69)$} & \multicolumn{2}{|c|}{ Sequencing, PTEN and pCR data available $(n=45)$} \\
\hline & No. of patients & $\%$ & No. of patients & $\%$ & No. of patients & $\%$ \\
\hline \multicolumn{7}{|l|}{ ER status } \\
\hline Negative & 35 & 39.8 & 28 & 40.6 & 19 & 42.2 \\
\hline Positive & 53 & 60.2 & 41 & 59.4 & 26 & 57.8 \\
\hline \multicolumn{7}{|l|}{ PR status } \\
\hline Negative & 54 & 61.4 & 39 & 56.5 & 29 & 64.4 \\
\hline Positive & 34 & 38.6 & 30 & 43.5 & 16 & 35.6 \\
\hline \multicolumn{7}{|l|}{$\mathrm{pCR}$} \\
\hline Yes & 38 & 43.2 & 32 & 46.4 & 21 & 46.7 \\
\hline No & 41 & 46.6 & 37 & 53.6 & 24 & 53.3 \\
\hline Unknown & 9 & 10.2 & & & & \\
\hline \multicolumn{7}{|l|}{ Targeted therapy } \\
\hline Trastuzumab & 38 & 43.2 & 30 & 43.5 & 21 & 46.7 \\
\hline Lapatinib & 10 & 11.4 & 10 & 14.5 & 6 & 13.3 \\
\hline Trastuzumab + lapatinib & 40 & 45.4 & 29 & 42 & 18 & 40 \\
\hline \multicolumn{7}{|l|}{ Age, years } \\
\hline$<49$ & 41 & 46.6 & 36 & 52.2 & 25 & 55.6 \\
\hline$\geq 49$ & 47 & 53.4 & 33 & 47.8 & 20 & 44.4 \\
\hline \multicolumn{7}{|l|}{ Tumour size, $\mathrm{cm}$} \\
\hline$\leq 5$ & 56 & 63.6 & 46 & 66.7 & 30 & 66.7 \\
\hline$>5$ & 23 & 26.1 & 17 & 24.6 & 12 & 26.6 \\
\hline Unknown & 9 & 10.3 & 6 & 8.7 & 3 & 6.7 \\
\hline \multicolumn{7}{|l|}{ N stage } \\
\hline NO & 25 & 28.4 & 20 & 29 & 9 & 20 \\
\hline $\mathrm{N} 1$ & 50 & 56.8 & 40 & 58 & 31 & 68.9 \\
\hline N2 & 2 & 2.3 & 1 & 1.4 & 1 & 2.2 \\
\hline$N X$ & 2 & 2.3 & 2 & 2.9 & 1 & 2.2 \\
\hline Unknown & 9 & 10.2 & 6 & 8.7 & 3 & 6.7 \\
\hline \multicolumn{7}{|l|}{ M stage } \\
\hline MO & 88 & 100 & 69 & 100 & 45 & 100 \\
\hline \multicolumn{7}{|l|}{ Overall stage } \\
\hline$\| \mathrm{A}$ & 30 & 34.1 & 26 & 37.7 & 12 & 26.6 \\
\hline$\| B$ & 31 & 35.2 & 25 & 36.2 & 21 & 46.7 \\
\hline$\| \mathrm{A}$ & 7 & 8 & 4 & 5.8 & 3 & 6.7 \\
\hline$\| I \mid B$ & 10 & 11.4 & 8 & 11.6 & 6 & 13.3 \\
\hline$\| I C$ & 1 & 1.1 & 0 & 0 & 0 & 0 \\
\hline Unknown & 9 & 10.2 & 6 & 8.7 & 3 & 6.7 \\
\hline
\end{tabular}

Abbreviations: ER Oestrogen receptor, PR Progesterone receptor, $p C R$, Pathological complete response; PTEN Phosphatase and tensin homolog, TCHL Docetaxel, carboplatin, trastuzumab, lapatinib

A total of 88 patients were enrolled in the TCHL phase II neoadjuvant study. After quality control, 74 patient samples were genotyped. Of these 74 samples, pCR data were available for 69 patients. Forty-five tumour samples had sufficient material for PTEN analysis. The clinical characteristics of these three patient groups are shown

the TCH and TCHL arms (Fig. 4). There was no difference in pCR rates in patients with either PIK3CA-mutated tumours or ERBB family-mutated tumours compared with patients with WT tumours in either the TCH or the TCHL arm (Fig. 4a and b); however, when we combined PIK3CA and ERBB family mutations, in the TCHL arm, the pCR rate was higher for 9 patients whose tumours harboured a PIK3CA and/or an $E R B B$ family mutation compared with 20 patients with WT tumours $(77.8 \%$ vs. $35 \% ; p=0.05)$ (Fig. 4 c). 


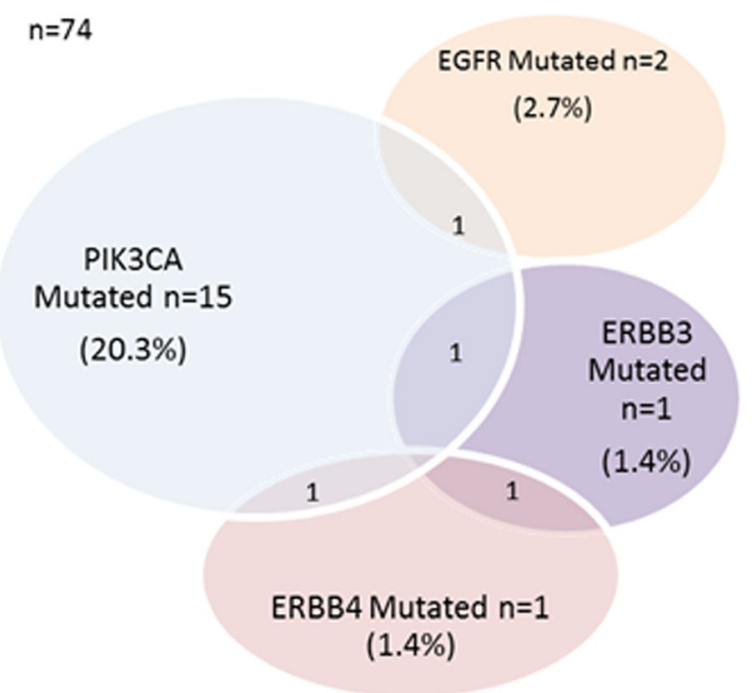

Fig. 1 Frequency of somatic PIK3CA and/or ERBB family mutations in baseline tumour biopsies from 74 patients enrolled in the TCHL clinical trial where adequate tumour was available following quality control. EGFR Epidermal growth factor receptor, ERBB3 Erb-B2 receptor tyrosine kinase 3, ERBB4 Erb-B2 receptor tyrosine kinase 4

There was no difference in $\mathrm{pCR}$ rates between these two groups of breast tumours in the $\mathrm{TCH}$ arm. The results shown in Fig. 4c suggest that a trend may exist in the data towards a benefit of TCHL treatment in patients with PIK3CA and/or ERBB family mutations, and although this was not significant after multiple

Table 2 Somatic mutation genotyping results by oestrogen receptor and progesterone receptor status and treatment arm

\begin{tabular}{|c|c|c|c|c|}
\hline & & $\begin{array}{l}\text { PIK3CA } \\
\text { mutated }\end{array}$ & $\begin{array}{l}\text { ERBB } \\
\text { mutated }\end{array}$ & $\begin{array}{l}\text { PIK3CA/ERBB } \\
\text { mutated }^{a}\end{array}$ \\
\hline $\begin{array}{l}\text { Tumour } \\
\text { group }\end{array}$ & $\begin{array}{l}\text { Total no. of } \\
\text { tumours }\end{array}$ & $\begin{array}{l}\text { No. of } \\
\text { tumours (\%) }\end{array}$ & $\begin{array}{l}\text { No. of } \\
\text { tumours (\%) }\end{array}$ & $\begin{array}{l}\text { No. of } \\
\text { tumours (\%) }\end{array}$ \\
\hline \multicolumn{5}{|l|}{ ER status } \\
\hline Negative & 29 & $8(27.6)$ & $1(3.4)$ & $8(27.6)$ \\
\hline Positive & 45 & $10(22.2)$ & $7(15.6)$ & 15 (33.3) \\
\hline \multicolumn{5}{|l|}{ PR status } \\
\hline Negative & 43 & $9(20.9)$ & $5(11.6)$ & 14 (32.6) \\
\hline Positive & 31 & $8(25.8)$ & $3(9.7)$ & $9(29)$ \\
\hline \multicolumn{5}{|c|}{ Treatment arm } \\
\hline $\mathrm{TCL}$ & 10 & $3(30)$ & $2(20)$ & $3(30)$ \\
\hline $\mathrm{TCH}$ & 32 & $9(28.1)$ & $2(6.3)$ & $10(31.3)$ \\
\hline TCHL & 32 & $6(18.8)$ & $4(12.5)$ & $10(31.3)$ \\
\hline
\end{tabular}

Abbreviations: $E R$ Oestrogen receptor, $P R$ Progesterone receptor, $T C H$ Docetaxel, carboplatin, and trastuzumab, TCHL Docetaxel, carboplatin, trastuzumab, lapatinib, TCL Docetaxel, carboplatin, lapatinib

Association of PIK3CA mutations, ERBB family mutations and combined PIK3CA $E R B B$ family mutations with ER and PR status. The mutation frequency in each treatment arm is also shown

${ }^{a}$ These numbers/percentages may not be a sum of PIK3CA and ERBB mutated because of mutation overlap testing (unadjusted $p$ value $=0.05$ ), it warrants further study in a larger cohort. When we included patients with low tumour expression of PTEN, there was no difference in pCR rates in patients whose tumours had a $P I K 3 C A / E R B B$ family mutation and/or low PTEN expression (i.e., PI3K activated) compared with patients with WT tumours in either the $\mathrm{TCH}$ or the TCHL arm (Fig. 4d and e).

Figure 4c shows that 9 of 29 tumours had PIK3CA or $E R B B$ mutations. Figure $4 \mathrm{~d}$ shows that 5 of 18 tumours had PTEN deficiency. Figure $4 \mathrm{e}$ shows that 8 of 18 tumours had mutations or PTEN deficiency, meaning that only 3 of the PIK3CA/ERBB-mutant tumours (from Fig. 4c) are included in the composite analysis in Fig. 4e.

\section{Discussion}

Biomarker analysis is necessary to identify those patients most likely to respond to neoadjuvant chemotherapy. In HER2-positive breast cancer, HER2 status is still the only recognised predictive marker to select patients for anti-HER2-targeted therapy; however, in recent years, a number of other biomarkers have emerged as potential predictors for response. PI3K pathway activation is the most common signal transduction pathway alteration in breast cancer $[17,25]$. It mostly results from somatic PIK3CA mutations or PTEN loss [26], but it can also be a result of mutations in $E R B B$ family genes $[20,27]$. Although a number of studies have reported on PI3K pathway activation in HER2-positive breast cancer, they have been focused mainly on its impact on response to anti-HER2 therapy in metastatic disease or in the adjuvant setting $[18,28-30]$. In the present study, we sought to evaluate associations between activation of the PI3K pathway and the efficacy of trastuzumab and lapatinib therapy in the neoadjuvant setting in early HER2-positive breast cancer.

Whereas pre-clinical studies have shown that PI3K pathway activation contributes to resistance to antiHER2-targeted therapies [18, 31], clinical studies have failed to give a clear answer. In the Neoadjuvant Lapatinib and/or Trastuzumab Treatment Optimization Trial (NeoALTTO), tumours with PIK3CA mutations had lower pCR rates after treatment with neoadjuvant paclitaxel plus HER2-targeted therapy, with pCR rates decreasing from $34.5 \%$ in PIK3CA WT tumours to $21.3 \%$ in mutated tumours [32]. Results from two sequential neoadjuvant studies (NCT00133796 and NCT00206427) demonstrated that patients with tumours with low PTEN expression or PI3K pathway mutations were less likely to achieve a pCR after neoadjuvant trastuzumab/ docetaxel than patients with tumours with no PI3K pathway mutations or high PTEN expression (18.2\% vs. $66.7 \% ; p=0.015$ ) [33]. In the Chemotherapy, Herceptin 
A

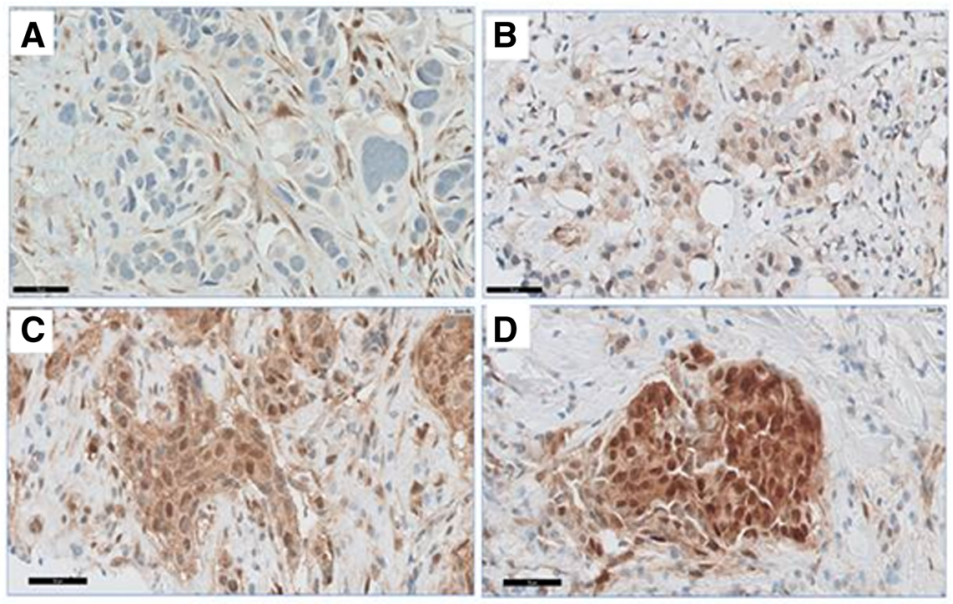

B
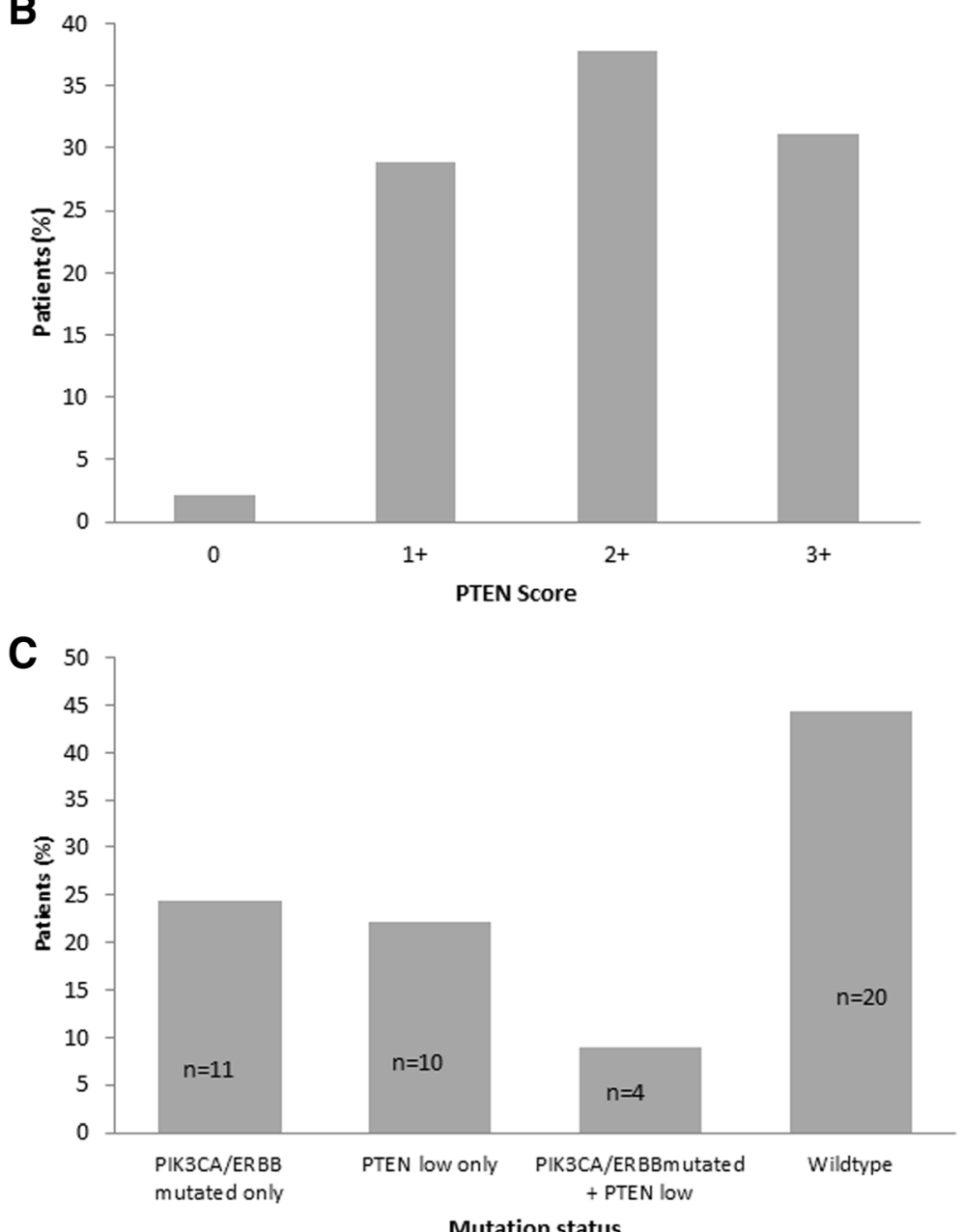

Mutation status

Fig. 2 a Immunohistochemical staining patterns of phosphatase and tensin homolog (PTEN). No immunostaining was detectable in breast carcinoma cells, but it was present in adjacent benign stromal cells $(0 ; A)$, cytoplasmic immunostaining weak $(1+; B)$, cytoplasmic immunostaining in carcinoma cells intermediate $(2+; C)$, cytoplasmic immunostaining strong $(3+; D)$ (scale bars $=50 \mu \mathrm{m})$. b Frequency of tumours with $0,1+, 2+$ and $3+$ immunostaining. All images were taken at $\times 40$ original magnification. $\mathbf{c}$ Frequency of PIK3CA/ERBB mutations, low expression of PTEN, and co-occurring PIK3CAVERBB mutations and low expression of PTEN (i.e., PI3K pathway activated) in 45 baseline tumour biopsies that were evaluable for PTEN expression 
Table 3 Somatic mutation genotyping and phosphatase and tensin homolog immunohistochemistry results by oestrogen receptor and progesterone receptor status and treatment arm

\begin{tabular}{llll}
\hline Patient group & $\begin{array}{l}\text { Total no. of } \\
\text { patients }\end{array}$ & $\begin{array}{l}\text { No. of } \\
\text { patients (\%) }\end{array}$ & $\begin{array}{l}\text { Po. of } \\
\text { patients (\%) }\end{array}$ \\
\hline $\begin{array}{llll}\text { ER status } \\
\text { Negative }\end{array}$ & 19 & $6(31.6)$ & $11(57.9)$ \\
$\begin{array}{l}\text { Positive } \\
\text { PR status }\end{array}$ & 26 & $8(30.8)$ & $14(53.8)$ \\
Negative & 29 & $11(37.9)$ & $17(58.6)$ \\
Positive & 16 & $3(18.8)$ & $8(50)$ \\
Treatment arm & & $2(33.3)$ & $4(66.7)$ \\
TCL & 6 & $7(33.3)$ & $13(61.9)$ \\
TCH & 21 & $5(31.3)$ & $8(44.4)$ \\
TCHL & 18 & & \\
\hline
\end{tabular}

Abbreviations: ER Oestrogen receptor, $P R$ Progesterone receptor, $T C H$ Docetaxel, carboplatin, and trastuzumab, TCHL Docetaxel, carboplatin, trastuzumab, lapatinib, TCL Docetaxel, carboplatin, lapatinib Association of low PTEN expression and combined low PTEN expression and PIK3CA/ERBB family mutations (i.e., PI3K pathway activation) with ER and PR status. The mutation frequency in each treatment arm is also shown

${ }^{a}$ Because of mutation overlap, these numbers/percentages may not be a sum of PIK3CA and ERBB mutated and Lapatinib in Operable Breast cancer (CHER-LOB) study, similar pCR rates after neoadjuvant paclitaxel for 12 weeks followed by fluorouracil, epirubicin and cyclophosphamide for four courses every 3 weeks plus HER2-targeted therapy were seen in patients with PIK3CA-WT and PIK3CA-mutated breast cancers (33.3\% vs. $22.7 \%$; $p=0.323$ ) [34]. A recent meta-analysis of pooled data from five neoadjuvant clinical trials demonstrated lower pCR rates in the PIK3CA mutant cohort than in the WT cohort $(16.2 \%$ vs. $29.6 \% ; p<0.001)$ [35].

In the present study, somatic PIK3CA mutations were found in $24.3 \%$ of tumours, and $E R B B$ family mutations were found in $10.8 \%$ of tumours, with a similar distribution in ER-positive or ER-negative tumours, which confirms results of previous studies [17, 21, 22]. Reduced PTEN expression was found in $31.1 \%$ of tumours. Combining these aberrations which result in activation of the PI3K pathway, we find $55.6 \%$ of tumours with PI3K pathway activation (Fig. 2c). However, there was no correlation between activation of the PI3K pathway and response to anti-HER2 neoadjuvant therapy in all study patients as measured by pCR (Fig. 3). The rate of pCR was similar among tumours with activation of the PI3K pathway (defined as a PIK3CA mutation and/or low PTEN expression and/or an $E R B B$ family mutation) and among tumours without a PIK3CA or ERBB family mutation or low PTEN expression ( $44 \%$ vs. $50 \% ; p=0.769$ ) (Fig. 3).

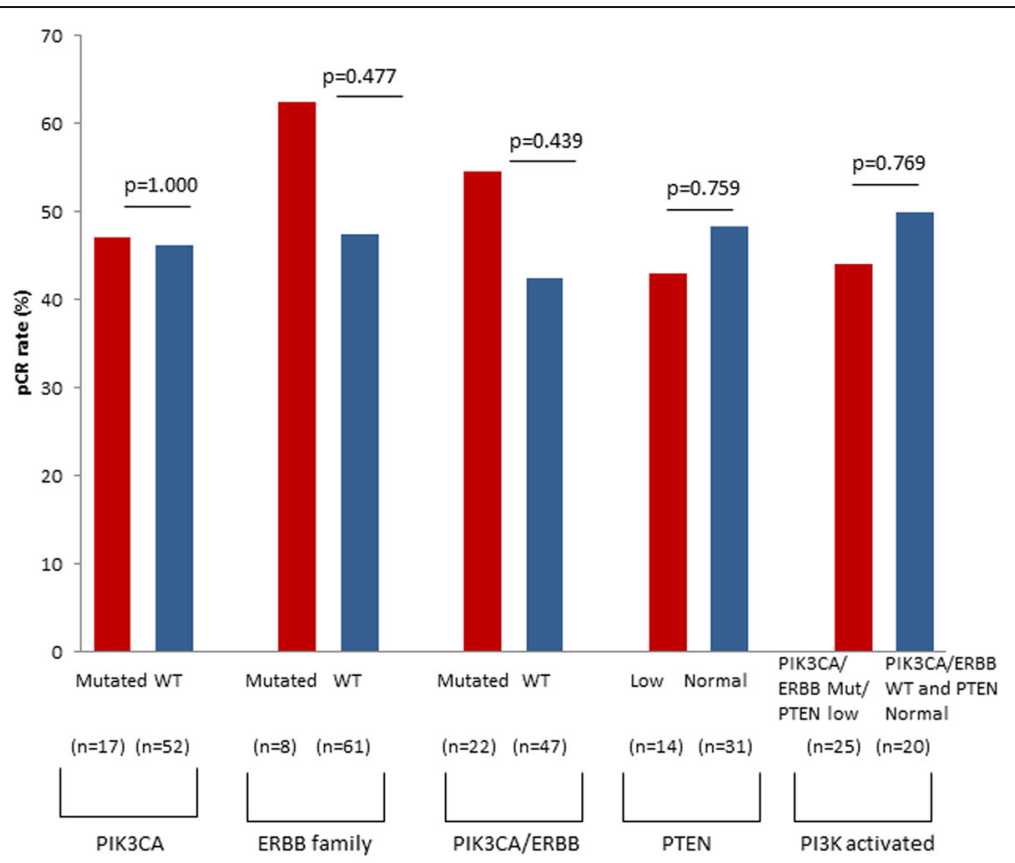

Fig. 3 Influence of PIK3CA and ERBB mutations and phosphatase and tensin homolog (PTEN) expression status on pathological complete response $(p C R)$ rates using a $x^{2}$ test of association. The proportion of patients who obtained $p C R$ is shown for the entire patient cohort. Phosphatidylinositol 3-kinase (PI3K) activation is defined as the presence of one or more of the following in a breast tumour: PIK3CA or ERBB family mutations or low PTEN expression. WT Wild type 
Table 4 Overall pathological complete response rates in the three treatment arms

\begin{tabular}{llll}
\hline Response & $\mathrm{TCL}(n=10)$ & $\mathrm{TCH}(n=36)$ & $\mathrm{TCHL}(n=33)$ \\
\hline $\mathrm{pCR}$ (no invasive cancer in the breast or LNs) & $2(20 \%)$ & $19(52.8 \%)$ & $17(51.5 \%)$ \\
$p=0.0839$ (TCL vs. TCH); 1.000 (TCH vs. TCHL); 0.1488 (TCL vs. TCHL) & & \\
\hline
\end{tabular}

Abbreviations: $L N$ Lymph node, $p C R$ Pathological complete response, $T C H$ Docetaxel, carboplatin, and trastuzumab, TCHL Docetaxel, carboplatin, trastuzumab, lapatinib, TCL Docetaxel, carboplatin, lapatinib

Overall $\mathrm{pCR}$ rates within each treatment arm following 6 cycles of neoadjuvant $\mathrm{TCL}$, TCH or TCHL

Pre-clinical studies suggest that PTEN loss may be a potential mechanism of resistance to HER2-targeted therapies [36, 37]. In the neoadjuvant setting, results are more variable. Dave et al. [33] showed that patients with low PTEN expression who received trastuzumab had lower pCR rates than those with high PTEN expression (15.4\% vs. $44.4 \%)$. However, in those patients who received lapatinib, low expression of PTEN was significantly associated with higher $\mathrm{pCR}$ rates than high expression (92.3\% vs. 41.2\%; $p=0.007)$. In the GeparQuattro study, low PTEN expression was also associated with lower response to trastuzumab-based chemotherapy compared with high PTEN expression (27.6\% vs. $57.1 \%$; $p=0.010$ ) [38]. In our present study, we did not find any association between PTEN expression and pCR, similar to the results of the NeoALTTO study [39]. The differences between the studies may be attributable to a lack of standardisation of PTEN detection and scoring methods, as well as to the lack of a standardised definition for low and high PTEN expression.

In the $\mathrm{TCH}$ arm, as in all study patients, the pCR rates were not significantly affected by PIK $3 C A$ or ERBB family mutations or by PTEN or PI3K activation status. In contrast, in the TCHL arm, patients with PIK3CA and/ or $E R B B$ family mutated tumours were more likely to achieve a pCR than patients with PIK3CA and ERBB WT tumours $(77.8 \%$ vs. $35 \% ; p=0.05)$ (Fig. 3). Our results are in contrast to NeoALTTO, where patients treated with a combination of weekly paclitaxel, trastuzumab and lapatinib who had PIK3CA-WT tumours had a pCR rate of $53.1 \%$, which decreased to $28.6 \%$ in patients with tumours harbouring PIK3CA mutations [32]. The difference in the impact of PIK3CA mutations in the two studies may be attributable to the low sample size in the TCHL cohort. Our study also included analysis of additional biomarkers of PI3K/AKT activation, including $E R B B$ family mutations, which suggests that a trend may exist within the data towards a benefit of TCHL treatment in patients with PIK3CA and/or ERBB family mutations, and this warrants further study in a larger cohort. Furthermore, it is worth noting that the neoadjuvant regimens used are different in the relevant study arms. TCHL for six cycles was used in our study versus HL (trastuzumab and lapatinib) followed by weekly paclitaxel for 12 weeks with $\mathrm{HL}$ in
NeoALLTO; therefore, sensitivity to the chemotherapy part of the regimen itself cannot be ruled out.

The finding that activating mutations in a signalling pathway can increase sensitivity to targeted therapies is not unusual. The tyrosine kinase inhibitors erlotinib and gefitinib show significant clinical responses in lung adenocarcinoma patients harbouring EGFR activating mutations [40], whereas dacomitinib, a pan-HER tyrosine kinase inhibitor has been shown to be beneficial in HER2-mutated lung tumours [41]. ERBB4 has been shown to be highly mutated in melanoma, where ERBB4 mutations increase ERBB4 kinase activity despite similar expression of the HER4 protein and sensitise cells to lapatinib [42]. In some, though not all, clinical studies, the combination of trastuzumab and lapatinib has been shown to be more effective than each drug given alone, likely because acquired drug resistance that results from the activation of alternate pro-survival pathways can be overcome by combination treatments [43]. In vitro, while PIK3CA mutated HER2-positive BT474 cells stably expressing the ERBB2-T798M mutation were resistant to trastuzumab, the addition of lapatinib or cetuximab restored sensitivity [44], likely through blocking heterodimer formation [44].

A limitation of our study and other studies is the absence of a group not treated with anti-HER2 therapy. Given that all patients received chemotherapy in association with anti-HER2 treatment, and also that there was considerable heterogeneity in the chemotherapy treatment regimens used between the studies, we cannot exclude the possibility that some of our findings are contributed to by sensitivity to the chemotherapy part of the regimen itself. We also acknowledge that our study is small, and it may be useful to validate these results in a larger cohort, where there will be a greater power to demonstrate a positive interaction between mutation status and pCR.

\section{Conclusions}

To the best of our knowledge this is the first study looking at the effects of PI3K pathway activation through a combination of PIK3CA gene mutations, $E R B B$ family gene mutations and low PTEN expression on pCR in the neoadjuvant treatment of HER2-positive breast cancer. Our study confirms the high prevalence of mutations 

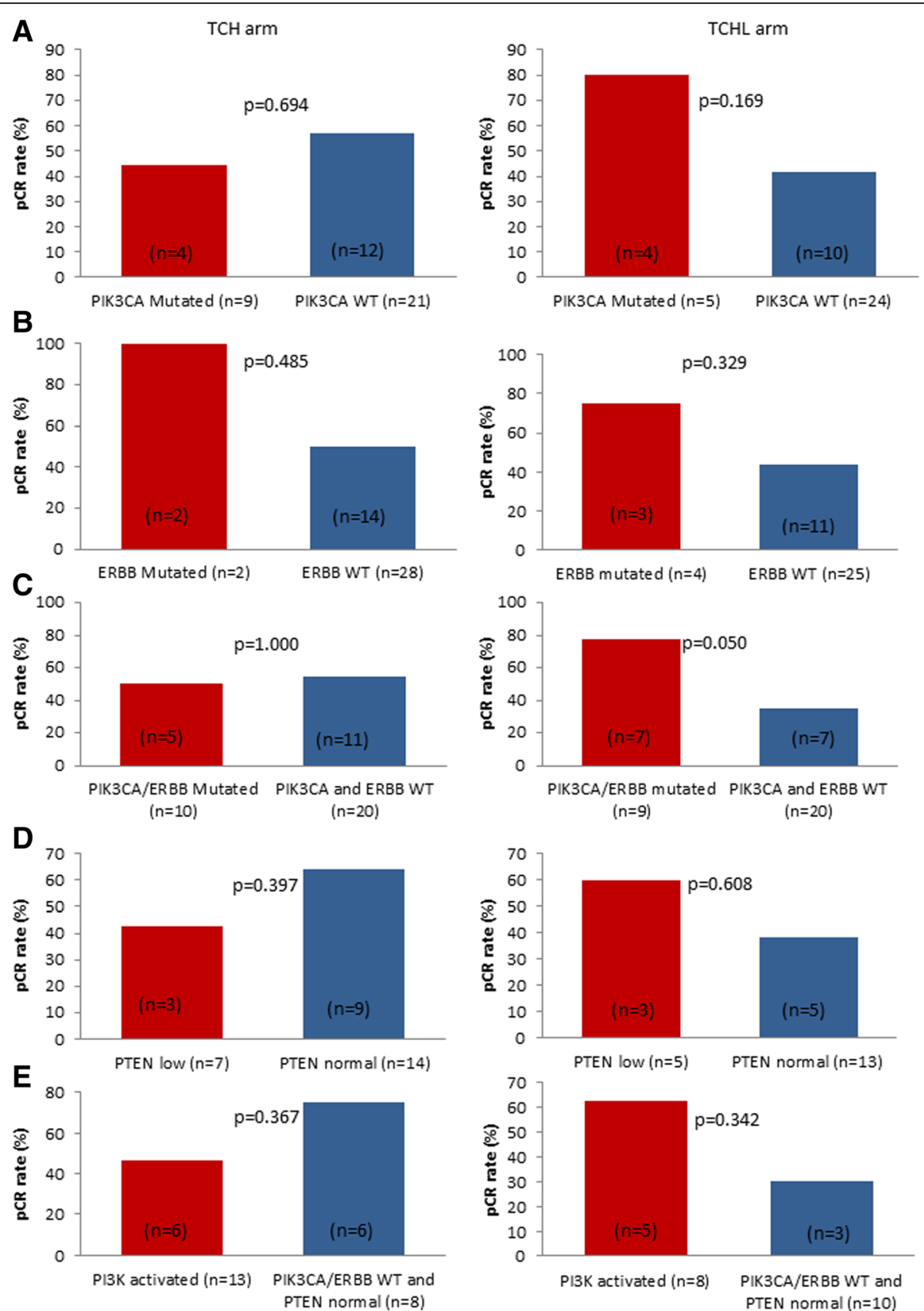

Fig. 4 Influence of mutation and phosphatase and tensin homolog (PTEN) status on pathological complete response (pCR) rate by treatment arm using a $x^{2}$ test of association. a Influence of PIK3CA mutations in tumours on pCR in patients receiving TCH (docetaxel, carboplatin, trastuzumab) and TCHL (docetaxel, carboplatin, trastuzumab, lapatinib). b Influence of ERBB family mutations in tumours on pCR in patients receiving TCH and TCHL. c Influence of combined PIK3CA and ERBB family mutation status in tumours on $\mathrm{PCR}$ in patients receiving TCH and TCHL. $\mathbf{d}$ Influence of low PTEN expression in breast tumours on pCR in patients receiving TCH and TCHL. e Influence of tumour phosphatidylinositol 3-kinase (PI3K) activation status on PCR in patients receiving TCH and TCHL. PI3K activation is defined as the presence of one or more of the following in a breast tumour: PIK3CA or ERBB family mutations or low PTEN expression. WT Wild type

that result in activation of the PI3K pathway in HER2positive breast cancer and their potential utility in predicting patients most likely to respond to combination anti-HER2 therapy. Because this is also the first report on the impact of PIK3CA mutations on responsiveness to neoadjuvant taxane plus carboplatin-based HER2targeted therapy regimens, with other studies such as NeoALLTO involving neoadjuvant taxane- or taxane/ anthracycline-based HER2-targeted therapy regimens, our findings need validation in other studies. 


\section{Additional files}

Additional file 1: Table S1. Non-synonymous somatic mutations in PIK3CA and ERBB family genes. (DOCX $15 \mathrm{~kb}$ )

Additional file 2: Table S2. Specific somatic mutations in PIK3CA, EGFR ERBB3, ERBB4 and PIK3CA detected in our patient cohort. (DOCX $13 \mathrm{~kb}$ )

\begin{abstract}
Abbreviations
ALTTO: Adjuvant Lapatinib and/or Trastuzumab Treatment Optimisation; CHER-LOB: Chemotherapy, Herceptin and Lapatinib in Operable Breast cancer study; DAB: 3,3'-Diaminobenzidine; EGFR: Epidermal growth factor receptor; ER: Oestrogen receptor; ERBB2: Receptor tyrosine-protein kinase erbB-2; ERBB3: Erb-B2 receptor tyrosine kinase 3; ERBB4: Erb-B2 receptor tyrosine kinase 4; FFPE: Fixed in formalin and embedded in paraffin wax; HER2: Human epidermal growth factor receptor 2; LN: Lymph node; NeoALTTO: Neoadjuvant Lapatinib and/or Trastuzumab Treatment Optimization Trial; pCR: Pathological complete response; PI3K: Phosphatidylinositol 3-kinase; PR: Progesterone receptor; PTEN: Phosphatase and tensin homolog; TCH: Docetaxel, carboplatin, trastuzumab; TCHL: Docetaxel, carboplatin, trastuzumab, lapatinib; TCL: Docetaxel, carboplatin, lapatinib; WT: Wild type
\end{abstract}

\section{Acknowledgements}

The TCHL study was sponsored by Cancer Trials Ireland (formerly known as the Irish Clinical Oncology Research Group [ICORG]), which received funding from GlaxoSmithKline.

\section{Funding}

This work was supported by the Irish Cancer Society Collaborative Cancer Research Centre under BREAST-PREDICT grant CCRC13GAL (www.breastpredict.com), the Health Research Board (HRA/POR2012/054), the North East Cancer Research and Education Trust, and the Science Foundation Ireland-funded Molecular Therapeutics for Cancer Ireland (08-SRC-B1410).

\section{Availability of data and materials}

Not applicable.

\section{Authors' contributions}

ST, AJE, JC and BTH conceived of and designed the study. ST, AJE, JF, KMS, AC and MM developed the study methodology. ST, AJE, JF, KMS, AC, MM, AT, EWK, NO'D, WG, LG, OB, JW, CK, BM, JK, GG, ADH, CP, DD and NH acquired data. ST, AJE, SFM, $J C$ and BTH analysed and interpreted data. ST, AJE, JF, KMS, AC, MM, AT, EWK, NO'D, WG, LG, OB, JW, CK, BM, JK, GG, AH, CP, DD, NH, JC and BTH wrote, reviewed and/ or revised the manuscript. All authors read and approved the final manuscript.

\section{Ethics approval and consent to participate}

In this study, we used samples that were collected under clinical trial protocols (ICORG 10-05; ClinicalTrials.gov, NCT01485926). Standardised ICORG procedures were used to acquire ethical approval for these studies. At the time of recruitment, patients were given an information leaflet and a consent form for storage and collection of biological materials, including blood and tissue samples, as well as future use of their samples for research purposes. The study protocol was approved by the institutional review boards of St. James's Hospital, Dublin; St. Vincent's University Hospital, Dublin; Bon Secours Hospital, Cork; Cork University Hospital; Beaumont Hospital, Dublin; Mater Misericordiae University and Private Hospitals, Dublin; Galway University Hospital, Galway; Letterkenny General Hospital, Letterkenny; Mid-Western Regional Hospital, Limerick; Sligo General Hospital, Sligo; and Waterford Regional Hospital, Waterford.

\section{Consent for publication}

Not applicable.

\section{Competing interests}

The authors declare that they have no competing interests.

\section{Publisher's Note}

Springer Nature remains neutral with regard to jurisdictional claims in published maps and institutional affiliations.

\section{Author details}

Medical Oncology Group, Department of Molecular Medicine, Royal College of Surgeons in Ireland, Dublin 9, Ireland. Department of Histopathology, Royal College of Surgeons in Ireland, Dublin, Ireland. ${ }^{3}$ Cancer Trials Ireland, Dublin, Ireland. ${ }^{4}$ National Institute for Cellular Biotechnology, Dublin City University, Dublin, Ireland. ${ }^{5}$ Cancer Biology and Therapeutics Laboratory, UCD School of Biomolecular and Biomedical Science, UCD Conway Institute, University College Dublin, Dublin, Ireland. ${ }^{6}$ Department of Medical Oncology, Beaumont Hospital, Dublin, Ireland. ${ }^{7}$ Department of Medical Oncology, St. Vincent's University Hospital, Dublin, Ireland. ${ }^{8}$ Department of Medical Oncology, The Mater Misericordiae University Hospital, Dublin, Ireland. ${ }^{9}$ Department of Medical Oncology, St. James Hospital, Dublin, Ireland. ${ }^{10}$ Department of Surgery, Royal College of Surgeons In Ireland, Dublin, Ireland. ${ }^{11}$ Department of Radiology, Beaumont Hospital, Dublin, Ireland.

Received: 21 April 2017 Accepted: 13 July 2017

Published online: 27 July 2017

\section{References}

1. Slamon DJ, Clark GM, Wong SG, Levin WJ, Ullrich A, McGuire WL. Human breast cancer: correlation of relapse and survival with amplification of the HER-2/neu oncogene. Science. 1987;235(4785):177-82.

2. Piccart-Gebhart MJ, Procter M, Leyland-Jones B, Goldhirsch A, Untch M, Smith I, et al. Trastuzumab after adjuvant chemotherapy in HER2-positive breast cancer. N Engl J Med. 2005;353(16):1659-72.

3. Hurvitz SA, Dirix L, Kocsis J, Bianchi GV, Lu J, Vinholes J, et al. Phase ॥ randomized study of trastuzumab emtansine versus trastuzumab plus docetaxel in patients with human epidermal growth factor receptor 2positive metastatic breast cancer. J Clin Oncol. 2013;31(9):1157-63.

4. Baselga J, Gelmon KA, Verma S, Wardley A, Conte P, Miles D, et al. Phase II trial of pertuzumab and trastuzumab in patients with human epidermal growth factor receptor 2-positive metastatic breast cancer that progressed during prior trastuzumab therapy. J Clin Oncol. 2010;28(7):1138-44.

5. Verma S, Miles D, Gianni L, Krop IE, Welslau M, Baselga J, et al. Trastuzumab emtansine for HER2-positive advanced breast cancer. N Engl J Med. 2012; 367(19):1783-91

6. Geyer CE, Forster J, Lindquist D, Chan S, Romieu CG, Pienkowski T, et al. Lapatinib plus capecitabine for HER2-positive advanced breast cancer. N Engl J Med. 2006;355(26):2733-43.

7. O'Donovan N, Byrne AT, O'Connor AE, McGee S, Gallagher WM, Crown J. Synergistic interaction between trastuzumab and EGFR/HER-2 tyrosine kinase inhibitors in HER-2 positive breast cancer cells. Investig New Drugs. 2011;29(5):752-9.

8. Guarneri V, Frassoldati A, Bottini A, Cagossi K, Bisagni G, Sarti S, et al. Preoperative chemotherapy plus trastuzumab, lapatinib, or both in human epidermal growth factor receptor 2-positive operable breast cancer: results of the randomized phase II CHER-LOB study. J Clin Oncol. 2012;30(16):1989-95.

9. Untch M, Loibl S, Bischoff J, Eidtmann H, Kaufmann M, Blohmer JU, et al. Lapatinib versus trastuzumab in combination with neoadjuvant anthracycline-taxane-based chemotherapy (GeparQuinto, GBG 44): a randomised phase 3 trial. Lancet Oncol. 2012;13(2):135-44.

10. von Minckwitz G, Schneeweiss A, Loibl S, Salat C, Denkert C, Rezai M, et al. Neoadjuvant carboplatin in patients with triple-negative and HER2-positive early breast cancer (GeparSixto; GBG 66): a randomised phase 2 trial. Lancet Oncol. 2014;15(7):747-56.

11. Baselga J, Bradbury I, Eidtmann H, Di Cosimo S, de Azambuja E, Aura C, et al. Lapatinib with trastuzumab for HER2-positive early breast cancer (NeoALTTO): a randomised, open-label, multicentre, phase 3 trial. Lancet. 2012:379(9816):633-40.

12. Robidoux A, Tang G, Rastogi P, Geyer Jr CE, Azar CA, Atkins JN, et al. Lapatinib as a component of neoadjuvant therapy for HER2-positive operable breast cancer (NSABP protocol B-41): an open-label, randomised phase 3 trial. Lancet Oncol. 2013;14(12):1183-92.

13. Bonnefoi H, Jacot W, Saghatchian M, Moldovan C, Venat-Bouvet L, Zaman K, et al. Neoadjuvant treatment with docetaxel plus lapatinib, trastuzumab, or both followed by an anthracycline-based chemotherapy in HER2-positive breast cancer: results of the randomised phase II EORTC 10054 study. Ann Oncol. 2015;26(2):325-32.

14. Crown JL, Keane M, Kennedy J, O'Reilly S, Kelly C, O'Connor M, et al. Randomized phase II study of pre-operative docetaxel, carboplatin with trastuzumab $(\mathrm{TCH})$ and/or/lapatinib $(\mathrm{L})$ in HER-2 positive $(\mathrm{H}+)$ breast cancer 
patients (BC pts). ICORG 10-05 [abstract]. Cancer Res. 2013;73(24 Suppl): Abstract P4-12-25. doi:10.1158/0008-5472.SABCS13-P4-12-25.

15. Hennessy BT, Smith DL, Ram PT, Lu Y, Mills GB. Exploiting the PIJK/AKT pathway for cancer drug discovery. Nat Rev Drug Discov. 2005;4(12): 988-1004.

16. Bachman KE, Argani P, Samuels Y, Silliman N, Ptak J, Szabo S, et al. The PIK3CA gene is mutated with high frequency in human breast cancers. Cancer Biol Ther. 2004;3(8):772-5. A published erratum appears in Cancer Biol Ther. 2005;4(2):133.

17. Stemke-Hale K, Gonzalez-Angulo AM, Lluch A, Neve RM, Kuo WL, Davies M, et al. An integrative genomic and proteomic analysis of PIK3CA, PTEN, and AKT mutations in breast cancer. Cancer Res. 2008;68(15):6084-91.

18. Berns K, Horlings HM, Hennessy BT, Madiredjo M, Hijmans EM, Beelen K, et al. A functional genetic approach identifies the PI3K pathway as a major determinant of trastuzumab resistance in breast cancer. Cancer Cell. 2007: 12(4):395-402

19. O'Brien NA, Browne BC, Chow L, Wang Y, Ginther C, Arboleda J, et al. Activated phosphoinositide 3-kinase/AKT signaling confers resistance to trastuzumab but not lapatinib. Mol Cancer Ther. 2010;9(6):1489-502.

20. Elster N, Collins DM, Toomey S, Crown J, Eustace AJ, Hennessy BT. HER2family signalling mechanisms, clinical implications and targeting in breast cancer. Breast Cancer Res Treat. 2015;149(1):5-15.

21. Gao J, Aksoy BA, Dogrusoz U, Dresdner G, Gross B, Sumer SO, et al. Integrative analysis of complex cancer genomics and clinical profiles using the cBioPortal. Sci Signal. 2013;6(269):11.

22. Cerami E, Gao J, Dogrusoz U, Gross BE, Sumer SO, Aksoy BA, et al. The cBio cancer genomics portal: an open platform for exploring multidimensional cancer genomics data. Cancer Discov. 2012;2(5):401-4.

23. Djordjevic B, Hennessy BT, Li J, Barkoh BA, Luthra R, Mills GB, et al. Clinical assessment of PTEN loss in endometrial carcinoma: immunohistochemistry outperforms gene sequencing. Mod Pathol. 2012;25(5):699-708.

24. Piccart-Gebhart M, Holmes E, Baselga J, de Azambuja E, Dueck AC, Viale G, et al. Adjuvant lapatinib and trastuzumab for early human epidermal growth factor receptor 2-positive breast cancer: results from the randomized phase III Adjuvant Lapatinib and/or Trastuzumab Treatment Optimization trial. J Clin Oncol. 2016;34(10):1034-42.

25. Engelman JA. Targeting PI3K signalling in cancer: opportunities, challenges and limitations. Nat Rev Cancer. 2009;9(8):550-62.

26. Brugge J, Hung MC, Mills GB. A new mutational AKTivation in the PI3K pathway. Cancer Cell. 2007;12(2):104-7.

27. Herter-Sprie GS, Greulich H, Wong KK. Activating mutations in ERBB2 and their impact on diagnostics and treatment. Front Oncol. 2013;3:86.

28. Jensen JD, Knoop A, Laenkholm AV, Grauslund M, Jensen MB, SantoniRugiu E, et al. PIK3CA mutations, PTEN, and pHER2 expression and impact on outcome in HER2-positive early-stage breast cancer patients treated with adjuvant chemotherapy and trastuzumab. Ann Oncol. 2012; 23(8):2034-42.

29. Loi S, Michiels S, Lambrechts D, Fumagalli D, Claes B, KellokumpuLehtinen $\mathrm{PL}$, et al. Somatic mutation profiling and associations with prognosis and trastuzumab benefit in early breast cancer. J Natl Cancer Inst. 2013;105(13):960-7.

30. Chandarlapaty S, Sakr RA, Giri D, Patil S, Heguy A, Morrow M, et al. Frequent mutational activation of the PI3K-AKT pathway in trastuzumab-resistant breast cancer. Clin Cancer Res. 2012;18(24):6784-91.

31. Elster N, Cremona M, Morgan C, Toomey S, Carr A, O'Grady A, et al. A preclinical evaluation of the PI3K alpha/delta dominant inhibitor BAY 806946 in HER2-positive breast cancer models with acquired resistance to the HER2-targeted therapies trastuzumab and lapatinib. Breast Cancer Res Treat. 2015;149(2):373-83.

32. Majewski IJ, Nuciforo P, Mittempergher L, Bosma AJ, Eidtmann H, Holmes E, et al. PIK3CA mutations are associated with decreased benefit to neoadjuvant human epidermal growth factor receptor 2-targeted therapies in breast cancer. J Clin Oncol. 2015;33(12):1334-9.

33. Dave B, Migliaccio I, Gutierrez MC, Wu MF, Chamness GC, Wong H, et al. Loss of phosphatase and tensin homolog or phosphoinositol-3 kinase activation and response to trastuzumab or lapatinib in human epidermal growth factor receptor 2-overexpressing locally advanced breast cancers. Clin Oncol. 2011;29(2):166-73.

34. Guarneri V, Dieci MV, Frassoldati A, Maiorana A, Ficarra G, Bettelli S, et al. Prospective biomarker analysis of the randomized CHER-LOB study evaluating the dual anti-HER2 treatment with trastuzumab and lapatinib plus chemotherapy as neoadjuvant therapy for HER2-positive breast cancer. Oncologist. 2015;20(9):1001-10.

35. Loibl S, Majewski I, Guarneri V, Nekljudova V, Holmes E, Bria E, et al. PIK3CA mutations are associated with reduced pathological complete response rates in primary HER2-positive breast cancer: pooled analysis of 967 patients from five prospective trials investigating lapatinib and trastuzumab. Ann Oncol. 2016;27(8):1519-25.

36. Eichhorn PJ, Gili M, Scaltriti M, Serra V, Guzman M, Nijkamp W, et al. Phosphatidylinositol 3-kinase hyperactivation results in lapatinib resistance that is reversed by the mTOR/phosphatidylinositol 3-kinase inhibitor NVPBEZ235. Cancer Res. 2008:68(22):9221-30.

37. Nagata Y, Lan KH, Zhou X, Tan M, Esteva FJ, Sahin AA, et al. PTEN activation contributes to tumor inhibition by trastuzumab, and loss of PTEN predicts trastuzumab resistance in patients. Cancer Cell. 2004;6(2):117-27.

38. Loibl S, Darb-Esfahani S, Huober J, Klimowicz A, Furlanetto J, Lederer B, et al. Integrated analysis of PTEN and p4EBP1 protein expression as predictors for pCR in HER2-positive breast cancer. Clin Cancer Res. 2016;22(11):2675-83.

39. Nuciforo PG, Aura C, Holmes E, Prudkin L, Jimenez J, Martinez P, et al. Benefit to neoadjuvant anti-human epidermal growth factor receptor 2 (HER2)-targeted therapies in HER2-positive primary breast cancer is independent of phosphatase and tensin homolog deleted from chromosome 10 (PTEN) status. Ann Oncol. 2015;26(7):1494-500.

40. Pao W, Miller V, Zakowski M, Doherty J, Politi K, Sarkaria I, et al. EGF receptor gene mutations are common in lung cancers from "never smokers" and are associated with sensitivity of tumors to gefitinib and erlotinib. Proc Natl Acad Sci U S A. 2004;101(36):13306-11.

41. Kris MG, Camidge DR, Giaccone G, Hida T, Li BT, O'Connell J, et al. Targeting HER2 aberrations as actionable drivers in lung cancers: phase II trial of the pan-HER tyrosine kinase inhibitor dacomitinib in patients with HER2-mutant or amplified tumors. Ann Oncol. 2015;26(7):1421-7.

42. Prickett TD, Agrawal NS, Wei X, Yates KE, Lin JC, Wunderlich JR, et al. Analysis of the tyrosine kinome in melanoma reveals recurrent mutations in ERBB4. Nat Genet. 2009:41(10):1127-32.

43. Garrett JT, Olivares MG, Rinehart C, Granja-Ingram ND, Sanchez V, Chakrabarty A, et al. Transcriptional and posttranslational up-regulation of HER3 (ErbB3) compensates for inhibition of the HER2 tyrosine kinase. Proc Natl Acad Sci U S A. 2011;108(12):5021-6.

44. Rexer BN, Ghosh R, Narasanna A, Estrada MV, Chakrabarty A, Song Y, et al. Human breast cancer cells harboring a gatekeeper T798M mutation in HER2 overexpress EGFR ligands and are sensitive to dual inhibition of EGFR and HER2. Clin Cancer Res. 2013:19(19):5390-401.

\section{Submit your next manuscript to BioMed Central and we will help you at every step:}

- We accept pre-submission inquiries

- Our selector tool helps you to find the most relevant journal

- We provide round the clock customer support

- Convenient online submission

- Thorough peer review

- Inclusion in PubMed and all major indexing services

- Maximum visibility for your research

Submit your manuscript at www.biomedcentral.com/submit
) Biomed Central 